\title{
Structural characterization of B-amyloid oligomer-aggregates by ion mobility mass spectrometry and electron spin resonance spectroscopy
}

\author{
Marius Ionuț Iuraşcu • Claudia Cozma • \\ Nick Tomczyk • John Rontree • Michael Desor • \\ Malte Drescher - Michael Przybylski
}

Received: 19 June 2009/Revised: 15 September 2009/Accepted: 16 September 2009/Published online: 17 October 2009

\begin{abstract}
Formation and accumulation of fibrillar plaques and aggregates of $B$-amyloid peptide $(A \beta)$ in brain have been recognized as characteristics of Alzheimer's disease (AD). Oligomeric aggregates of $A B$ are considered critical intermediates leading to progressive neurodegeneration; however, molecular details of the oligomerization and aggregation pathway and the structures of $\mathrm{AB}$-oligomers are hitherto unclear. Using an in vitro fibril formation procedure of $A B(1-40)$, $B$-amyloid aggregates were prepared and insoluble aggregates separated from soluble products by centrifugation. In this study, ion mobility mass
\end{abstract}

M. Ionuț Iuraşcu $\cdot$ C. Cozma $\cdot$ M. Przybylski

Laboratory of Analytical Chemistry and Biopolymer Structure

Analysis, Department of Chemistry, University of Konstanz,

78457 Konstanz, Germany

N. Tomczyk $\cdot$ J. Rontree $\cdot M$. Desor

Waters Corporation-MS Technologies Centre,

Atlas Park Simonsway,

Manchester M22 5PP, UK

N. Tomczyk $\cdot$ J. Rontree $\cdot$ M. Desor

Waters $\mathrm{GmbH}$,

Helfmann-Park 10,

65760 Eschborn, Germany

M. Drescher

Physical Chemistry, Department of Chemistry,

University of Konstanz,

78457 Konstanz, Germany

M. Drescher $(\square) \cdot$ M. Przybylski $(\square)$

Department of Chemistry, University of Konstanz, Universitätsstrasse 10 ,

78457 Konstanz, Germany

e-mail: Malte.Drescher@physik.uni-karlsruhe.de

e-mail: Michael.Przybylski@uni-konstanz.de spectrometry (IM-MS) was applied in combination with electron paramagnetic resonance spectroscopy (EPR) to the identification of the components of $A B$-oligomers, and to their structural and topographical characterization. The formation of $\mathrm{AB}$-oligomers and aggregates was monitored by gel electrophoresis, and Aß-oligomer bands were identified by in-gel tryptic digestion and matrix-assisted ' laser desorption ionization-mass spectrometry (MALDI-MS) to consist predominantly of $A B(1-40)$ peptide. First, ion mobility-MS studies of soluble $A ß$-aggregates prepared by incubation for 5 days were performed on a quadrupole timeof-flight mass spectrometer and revealed (1) the presence of at least two different conformational states, and (2), the formation of Met-35 oxidized products. For estimation of the size of $A B$-aggregates using EPR spectroscopy, a modified $A ß(1-40)$ peptide containing an additional $N$-terminal cysteine residue was prepared, and a 3-(2-iodoacetamido)2,2,5,5-tetramethyl-1-pyrrolidinyloxy radical spin label derivative (IPSL) was coupled by S-alkylation. The EPR spectra of the spin-labeled Cys-AB(1-40) oligomers were matched with spectra simulations using a multi-component simulation strategy, resulting in complete agreement with the gel electrophoresis results.

Keywords Alzheimer's disease - $B$-Amyloid - Aß-fibrils . Oligomerization - Ion mobility mass spectrometry .

EPR spectroscopy

\section{Introduction}

A significant proportion of elderly population is affected by progressive neurodegenerative disorders. Formation and accumulation of fibrillar plaques and aggregates of 
$\beta$-amyloid peptide $(A \beta)$ and $\alpha$-synuclein (Syn) in brain have been recognized as characteristics of Alzheimer's disease (AD) and Parkinson's disease (PD) [1-3]. AB is a polypeptide containing 39-43 amino acid residues derived from proteolytic cleavage of the transmembrane $A B$ precursor protein (APP). Recently, the formation of $A B$-oligomers has become of particular interest, since oligomers have been suggested to be key neurotoxic species for progressive neurodegeneration $[2,4,5]$; however, molecular details of the pathophysiological degradation of APP, and of AB-aggregation pathways are hitherto unclear [6]. Despite the lack of molecular mechanism(s), studies towards the development of immunotherapeutic approaches for $\mathrm{AD}$ [7] have shown initial success in the production of therapeutic antibodies that disaggregate $A ß$-fibrils and improve the memory impairment in transgenic AD mice [8-11]. The identification of the epitopes recognized by both $A B$-plaque specific and aggregation-preventing antibodies, and the discovery of their $A ß$-oligomer specificity are presently causing enhanced interest in the elucidation of $A B$-oligomeric structures [12-16].

Ion mobility mass spectrometry (IM-MS) is a new MS method that has received increasing attention as a tool for the characterization of molecular assemblies according to their conformation and/or topography [17-20]. The IM-MS instrument employed in this study consists of two parts, (a) an ion mobility drift cell where ions are separated within an electric field according to their collisional cross-section, and (b) a quadrupole time-of-flight mass spectrometer (Synapt-QTOF-MS) [21-23]. Thus, the IM-MS implements a new mode of separation that allows the differentiation of protein conformational states.

EPR spectroscopy is a technique for studying chemical species with unpaired electrons that has recently shown to be an efficient bioanalytical tool for determination of molecular distances and molecular size estimations from spin motion measurements [24-30]. This technique has recently shown high sensitivity compared to the traditional techniques for protein structure determination, such as NMR spectroscopy [31]. In this study, ion mobility-MS in combination with EPR was applied to the study of $A ß$-oligomers prepared in vitro from $A B(1-40)$. For $E P R$ studies of $A B$-oligomers and aggregates, $N$-cysteinyl-Aß(1-40) peptides were synthesized by solid phase peptide synthesis (SPPS) and an 3-(2iodoacetamido)-2,2,5,5-tetramethyl-1-pyrrolidinyloxy (iodo-proxyl) spin label derivative (IPSL) was attached to the N-terminal cysteine residue by alkylation. The complementary application of IM-MS and EPR provided first molecular details of $A \beta$-oligomers by the identification of oxidative structure modifications, and an estimation of the size and shape of $A B$-oligomers.

\section{Materials and methods}

Synthesis and purification of spin-labeled $A B$-peptide derivatives

Aß(1-40) (H-DAEFRHDSGYEVHHQKLVFFAEDVGSNK GAIIGLMVGGVV-NH $\left.{ }_{2}\right)$ and Cys-Aß(1-40) were synthesized by SPPS on a semiautomatic peptide synthesizer EPS 221 (Abimed/Intavis, Langenfeld, Germany), according to the Fmoc strategy. The synthesis was carried out on a NovaSyn ${ }^{(B)}$ TGR resin, with a loading capacity $0.33 \mathrm{mmol} / \mathrm{g}$. The spin label used was 3-(2iodoacetamido)-2,2,5,5-tetramethyl-1-pyrrolidinyloxy (Iodo-Proxyl; IPSL; Sigma-Alddich, München, Germany). The labeling reaction was performed using a tenfold molar excess of IPSL in $25 \mathrm{mM}$ Tris buffer, $\mathrm{pH} 9$, by incubation for $12 \mathrm{~h}$ at $4^{\circ} \mathrm{C}$ [32]. The synthetic $\dot{A} \beta$ peptides and the labeling reaction mixture were purified by reverse-phase high-performance liquid chromatography (RP-HPLC; Fig. 1) on a semi-preparative Vydac C4 column. The mobile phases were: $0.1 \%$ TFA in milliQ water (eluent $\mathrm{A}$ ) and $80 \%$ acetonitrile in eluent $\mathrm{A}$ (eluent $\mathrm{B}$ ); $\mathrm{a}$ linear gradient of $20-80 \%$ eluent $B$ was used in $30 \mathrm{~min}$ at a flow rate of $4 \mathrm{~mL} / \mathrm{min}$.

\section{Mass spectrometry}

Synthetic AB-peptides were separated by HPLC and analyzed by matrix-assisted laser desorption ionization time-of-flight mass spectrometry (MALDI-TOF MS) on a Bruker Daltonik Biflex ITM linear TOF mass spectrometer (Bruker Daltonik, Bremen, Germany). Aliquots of $0.8 \mu \mathrm{L}$ of peptide sample were mixed with $0.8 \mu \mathrm{L}$ 4-hydroxy $\alpha$-cyano cinnamic acid (HCCA) in AcCN:0.1\% TFA in water $2: 1(v: v)$. Upon lyophilization, peptide samples were redissolved in $0.2 \%$ formic acid at a concentration of $0.1 \mu \mathrm{g} / \mu \mathrm{L}$. HPLC was performed on an Agilent 1100 instrument (Agilent Technologies; Waldbronn, Germany) equipped with a C8 column (Vydac $150 \times 1 \mathrm{~mm}$, $5 \mu \mathrm{m}$ ), using a flow rate of $50 \mu 1 / \mathrm{min}$. The following gradient was used: equilibration step, $2 \% \mathrm{~B}$ for $5 \mathrm{~min}$, linear gradient from $2 \% \mathrm{~B}$ to $65 \% \mathrm{~B}$ in $63 \mathrm{~min}$; from $65 \%$ $\mathrm{B}$ to $98 \% \mathrm{~B}$ in $10 \mathrm{~min} ; 10 \mathrm{~min}$ washing step $98 \% \mathrm{~B}$; $10 \mathrm{~min}$ to return to the initial concentration of $2 \% \mathrm{~B}$ (solvent $\mathrm{A}, 0.2 \%$ formic acid in water, solvent $\mathrm{B}, 0.2 \%$ formic acid in acetonitrile). Electrospray mass spectrometry was performed on an Esquire $3000+$ ion trap spectrometer (Bruker Daltonik, Bremen, Germany) at the following conditions: capillary temperature $250^{\circ} \mathrm{C}$, nebulizer gas 20 psi (Ar), drying gas $9 \mathrm{l} / \mathrm{min}\left(\mathrm{N}_{2}\right)$, potential difference $4 \mathrm{kV}$ (positive-ion mode), endplate offset $500 \mathrm{~V}$, skimmer $40 \mathrm{~V}$, and capillary exit $136 \mathrm{~V}$. The ion trap was locked on automatic gain control, and six microscans were collected for 
Fig. 1 HPLC profiles (crude products) and MALDI-TOF mass spectra of HPLC-purified a Cys-AB(1-40) and b IPSLCys-Aß $(1-40)$ peptides
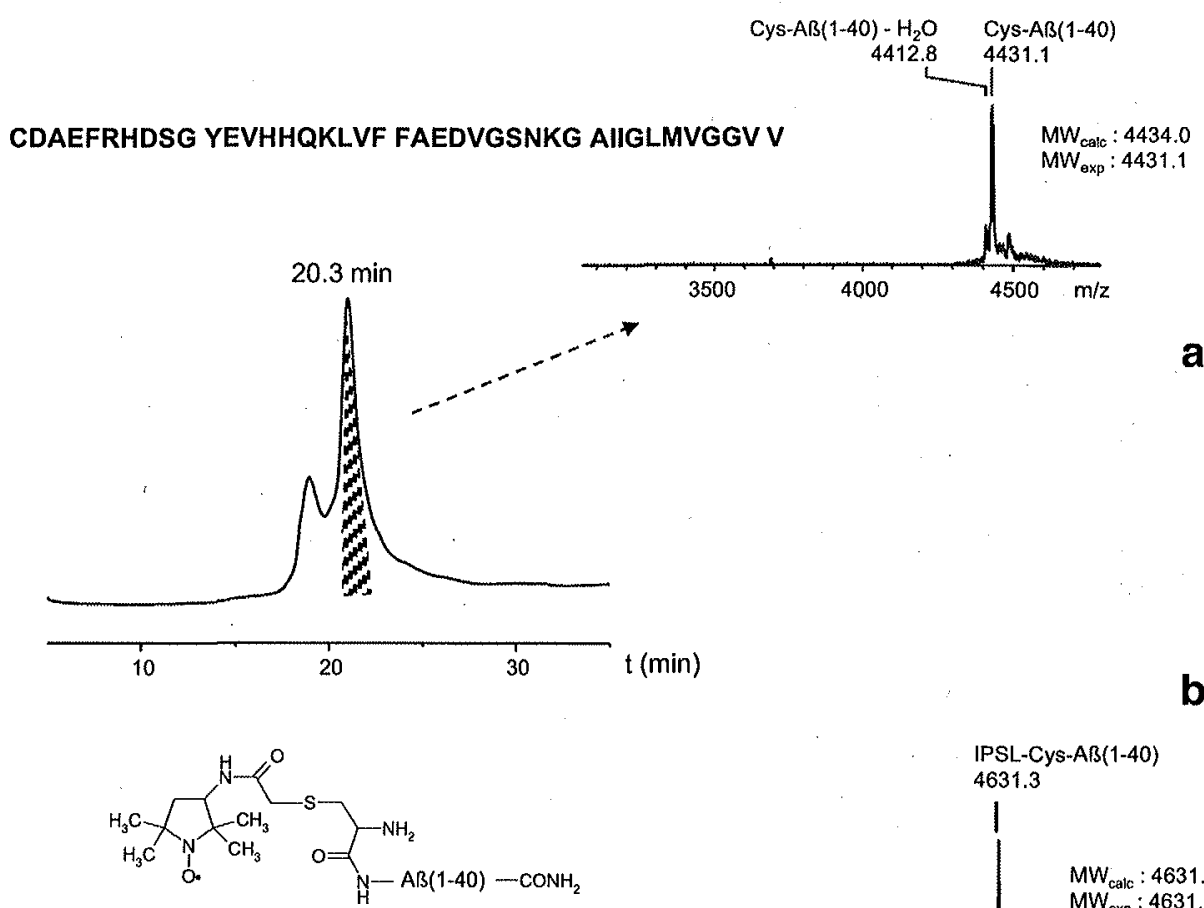

IPSL-Cys-Aß (1-40)

4631.3

$M W_{\text {calc }}: 4631.3$ $\mathrm{MW}_{\mathrm{exp}}: 4631.3$

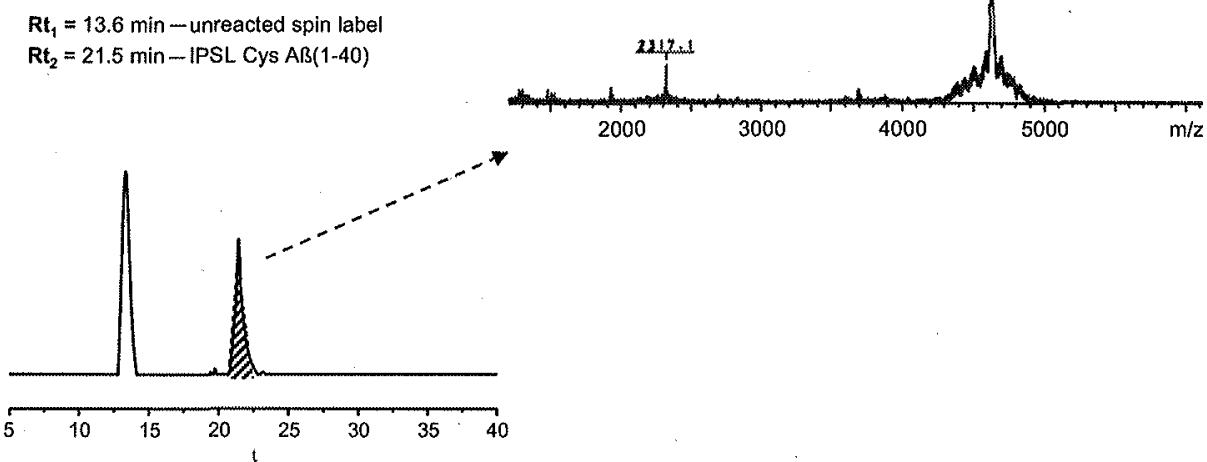

each full MS scan with a maximum accumulation time of $200 \mathrm{~ms}$ for each ion.

\section{Preparation of Aß-fibrils}

For $A ß$-oligomerization and fibril formation, $A B(1-40)$ and PSL-Cys-Aß $(1-40)$ peptides were solubilized at a concentration of $1 \mu \mathrm{g} / \mu \mathrm{L}$ in a buffer containing $50 \mathrm{mM} \mathrm{Na}_{3} \mathrm{PO}_{4}$, $150 \mathrm{mM} \mathrm{NaCl}, 0.02 \% \mathrm{NaN}_{3}$ at $\mathrm{pH} 7.5$ [30]. Because of the low solubility of Aß-fibrils, several sonication/vortex cycles were performed to ensure complete solubilization. Each solution was incubated for 5 days at $37^{\circ} \mathrm{C}$, yielding a white precipitate at the end of the incubation period. The precipitate was briefly sonicated, centrifuged $15 \mathrm{~min}$ at $13,000 \mathrm{rpm}$, and the supernatant removed and replaced with an equal volume of deionized water (MilliQ). Both the supernatant and the resuspended precipitate were subjected to gel electrophoresis and EPR spectroscopy experiments.

Gel electrophoresis

For in-gel proteolytic digestion and mass spectrometry, the resuspended precipitate fraction was subjected to SDSPAGE separation. The fibril fraction $(10 \mu \mathrm{L})$ was mixed with $10 \mu \mathrm{L}$ sample buffer, 4\% SDS, $25 \%$ glycerol, and bromophenol blue staining reagent. After loading on a $15 \%$ gel, electrophoresis was developed for $15 \mathrm{~min}$ at $60 \mathrm{~V}$, and subsequently for $1-2 \mathrm{~h}$ at $100 \mathrm{~V}$.

Gel electrophoresis of the spin-labeled IPSL-Cys$\mathrm{A} B(1-40)$ fibril preparation was carried out with a freshly prepared solution in fibril growth buffer, and a sample after incubation for 5 days (supernatant and 
resuspended precipitate). 1D Tris-tricine polyacrylamide gel electrophoresis (15\%) was run as described above and visualized by Coomassie blue staining.

In-gel tryptic digestion and peptide extraction

Protein spots were cut from the gel (Fig. 2a, bands 1-4), washed with MilliQ water, and the gel pieces shaken for $30 \mathrm{~min}$ at $25^{\circ} \mathrm{C}$ with $60 \%$ acetonitrile to dehydrate the gel, and then dried in a Speed Vac centrifuge $(30 \mathrm{~min})$. Protein spots were destained by addition of $50 \mathrm{mM} \mathrm{NH}_{4} \mathrm{HCO}_{3}$ (15 min), dehydrated with $60 \%$ acetonitrile solution $(15 \mathrm{~min})$ and dried in a Speed Vac centrifuge $(30 \mathrm{~min})$.
The gel pieces were then swollen in digestion buffer (12.5 ng trypsin $/ \mu \mathrm{l} 50 \mathrm{mM} \mathrm{NH} \mathrm{NHCO}_{3}$ ) at $4^{\circ} \mathrm{C}$ (on ice) for $45 \mathrm{~min}$, and incubated at $37^{\circ} \mathrm{C}$ overnight $(12 \mathrm{~h})$. After removal of supernatant, peptide extraction was performed at $25^{\circ} \mathrm{C}$ with a solution of $60 \%$ acetonitrile, $0.1 \%$ TFA in MilliQ water (three steps of $1 \mathrm{~h}$ each). The tryptic digestion mixtures were analyzed by MALDI-TOF MS, and data were searched against the NCBInr protein database by means of the Mascot MSMS ion search engine.

Ion mobility mass spectrometry

Ion mobility mass spectrometry was performed with a Waters SYNAPT quadrupole time-of-flight (QTOF) mass
Fig. 2 ESI-mass spectra (LC-MS) of a $A B(1-40)$, b Cys-AB(1-40), and $\mathrm{c}$ IPSL-Cys-Aß $(1-40)$

\section{DAEFRHDSGYEVHHQKLVFFAEDVGSNKGAIIGLMVGGW}

a

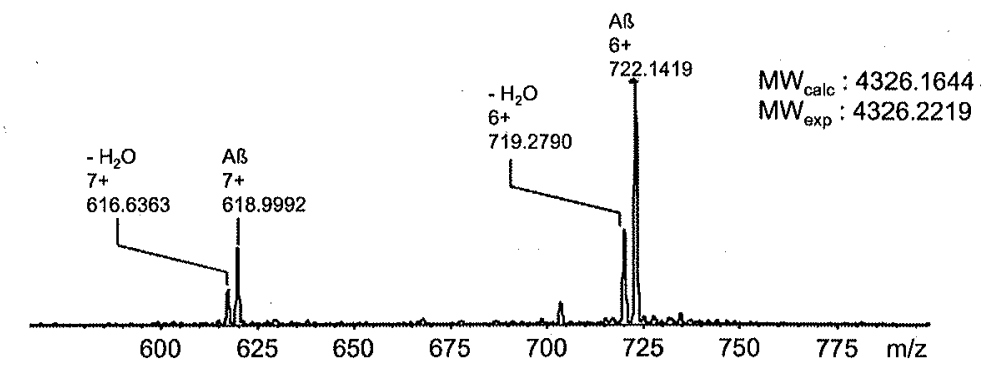

CDAEFRHDSGYEVHHQKLVFFAEDVGSNKGAIIGLMVGGVV

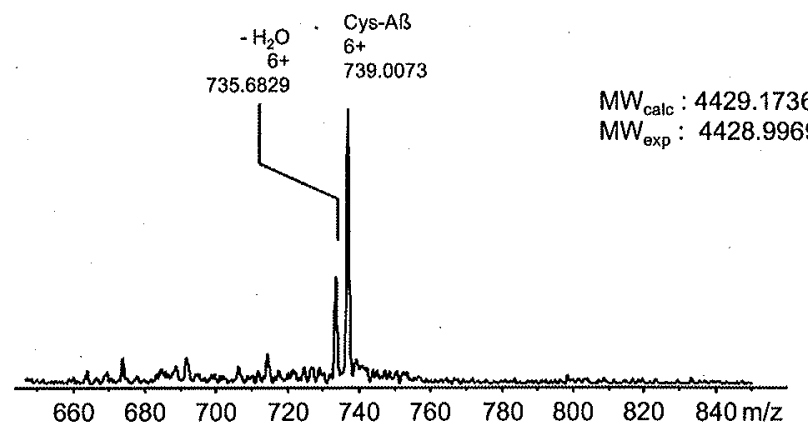

CDAEFRHDSGYEVHHQKLVFFAEDVGSNKGAIIGLMVGGVV IPSL

IPSL-Cys-Aß 772.1584

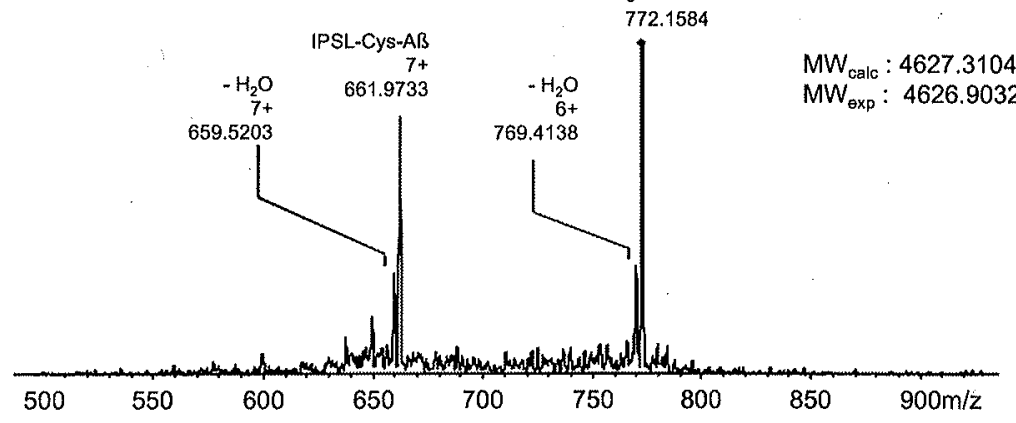


Fig. 3 1D-Gel electrophoresis (Coomassie blue staining) of $A ß$-aggregates in vitro. a SDSPAGE of $A B(1-40)$ after 5 days incubation at $37^{\circ} \mathrm{C}$; band $I$ $\mathrm{A} ß(1-40)$ monomer, band 2 $\mathrm{A} B$-oligomer, bands 3 and 4 high-molecular-weight aggregates ("soluble protofibrils"); bands 3 and 4 being only partially separated. b. Tris-tricine PAGE of IPSL-Cys-AB(1-40) after 5 days incubation at $37^{\circ} \mathrm{C}$. Lane $I$ a freshly prepared IPSLCys- $A B(1-40)$ solution (in fibril growth buffer), lane 2 precipitate after 5 days incubation; only the soluble part entered the separation gel, the rest remaining in the stacking gel, lane 3 supernatant after 5 days incubation. In band $l$ the monomeric IPSL-Cys-AB(1-40) is present, in band 2 the dimer, in band 3 the trimer, and in band 4 highmolecular-weight aggregates ("soluble protofibrils")
Table 1 Identification of $A B$-fragments in oligomer bands separated by gel electrophoresis (cf. Fig. 3a) by MALDITOF-MS

\section{LMW (kDa)}

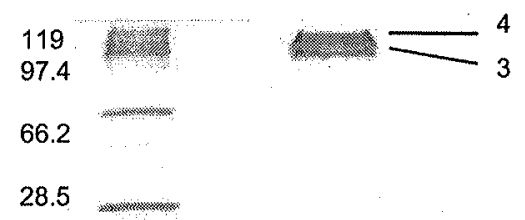

18.4

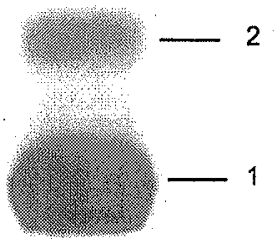

5

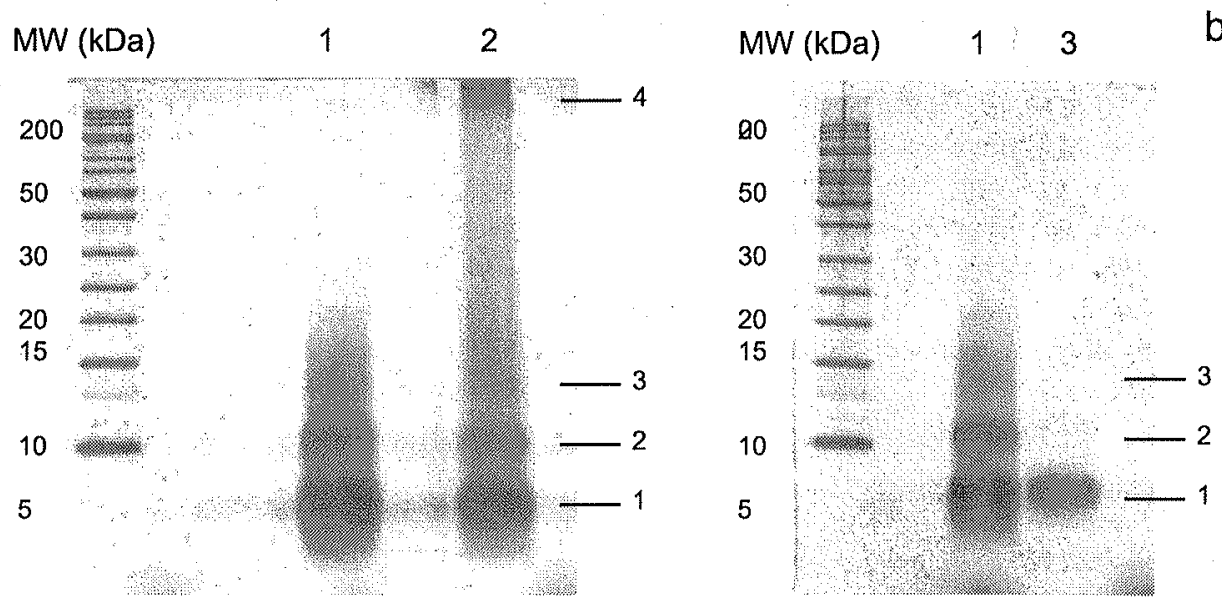
3 2 1

$b$

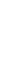

\section{.}


spectrometer (Waters Corp., Manchester, UK). A freshly prepared $A B(1-40)$ peptide solution $(1 \mu \mathrm{g} / \mu \mathrm{L})$, and the -supernatant fraction from in vitro fibril preparation ( 5 days incubation at $37^{\circ} \mathrm{C}$ ) were analyzed by injection of $5 \mu \mathrm{L}$ $(0.5 \mu \mathrm{g} / \mu \mathrm{L})$ in a desalting cartridge, and elution for $10 \mathrm{~min}$ at $20 \mu \mathrm{L} / \mathrm{min}$ with a gradient of acetonitrile from $10 \%$ to $90 \%$. Ion mobility-MS was performed in the $\mathrm{m} / \mathrm{z}$ range of $350-4,000$ at a pressure of $0.45 \mathrm{bar}$, cone voltage of $25 \mathrm{~V}$, and a drift voltage (wave height) of 5 to $15 \mathrm{~V}$.

\section{Electron paramagnetic resonance spectroscopy}

The freshly prepared IPSL-Cys-Aß(1-40) solution (in fibril growth buffer), and a sample incubated for 5 days as described above (precipitate and supernatant) were used for EPR spectroscopy. EPR spectra were obtained on a MiniScope MS200 (Magnettech GmbH, Berlin, Germany) spectrometer at $25^{\circ} \mathrm{C}$. An accumulation of 60 scans was carried out at $1 \mathrm{~min} / \mathrm{scan}$; the modulation amplitude was set at $400 \mathrm{G}$ and the attenuation at $23 \mathrm{~dB}$. Following acquisition, the spectra were fitted by simulations using the EasySpin package [33] and Matlab (The MathWorks Inc., Natick, Massachusetts, United States) software. From these simulations, multiple fractions featuring significantly
Fig. 5 Ion mobility-MS analysis of a freshly prepared $A B(1-40)$, b supernatant after 5 days of incubation; $\mathbf{c}$, $\mathbf{d}$ extracted ion mobility profiles for $m / z 866.6,[\mathrm{M}+5 \mathrm{H}]^{5+}$ from freshly prepared $A B(1-40)$ and supernatant

different correlation times were extracted. For all of these fractions, the following simulation parameters were kept constant: $g$-tensor $g=\left[\begin{array}{lll}2.01 & 2.0068 & 2.0027\end{array}\right]$, hyperfine tensor $A=\left[\begin{array}{lll}13 & 13 & 108.4\end{array}\right] \mathrm{MHz}$; and intrinsic linewidth $0.14 \mathrm{mT}$.

\section{Results and discussion}

Synthesis and characterization of spin-labeled $A ß-p e p t i d e s$

$\mathrm{AB}(1-40)$ and $N$-cysteinyl-Aß $(1-40)$ peptides were synthesized by SPPS at the $100 \mu \mathrm{M}$ scale according to the Fmoc strategy, and the crude products purified by RPHPLC (Fig. 1). AB(1-40) was separated at a retention time of $21.3 \mathrm{~min}$ at the HPLC conditions employed, while the Cys- $A B(1-40)$ derivative was isolated with "a slightly different retention time of $20.5 \mathrm{~min}$ (Fig. la). For the preparation of the IPSL-spin-labeled peptide derivative (see Fig. 1b), the Cys-Aß(1-40) crude product was used
Fig. 4 MALDI-MS of in-gel tryptic digestion mixture of $A B(1-40)$, high-molecularweight aggregates (band 4 from Fig. 2a). a MALDI-TOF mass spectrum of tryptic digestion mixture; $\mathbf{b}$ database search result with identified peptide fragments
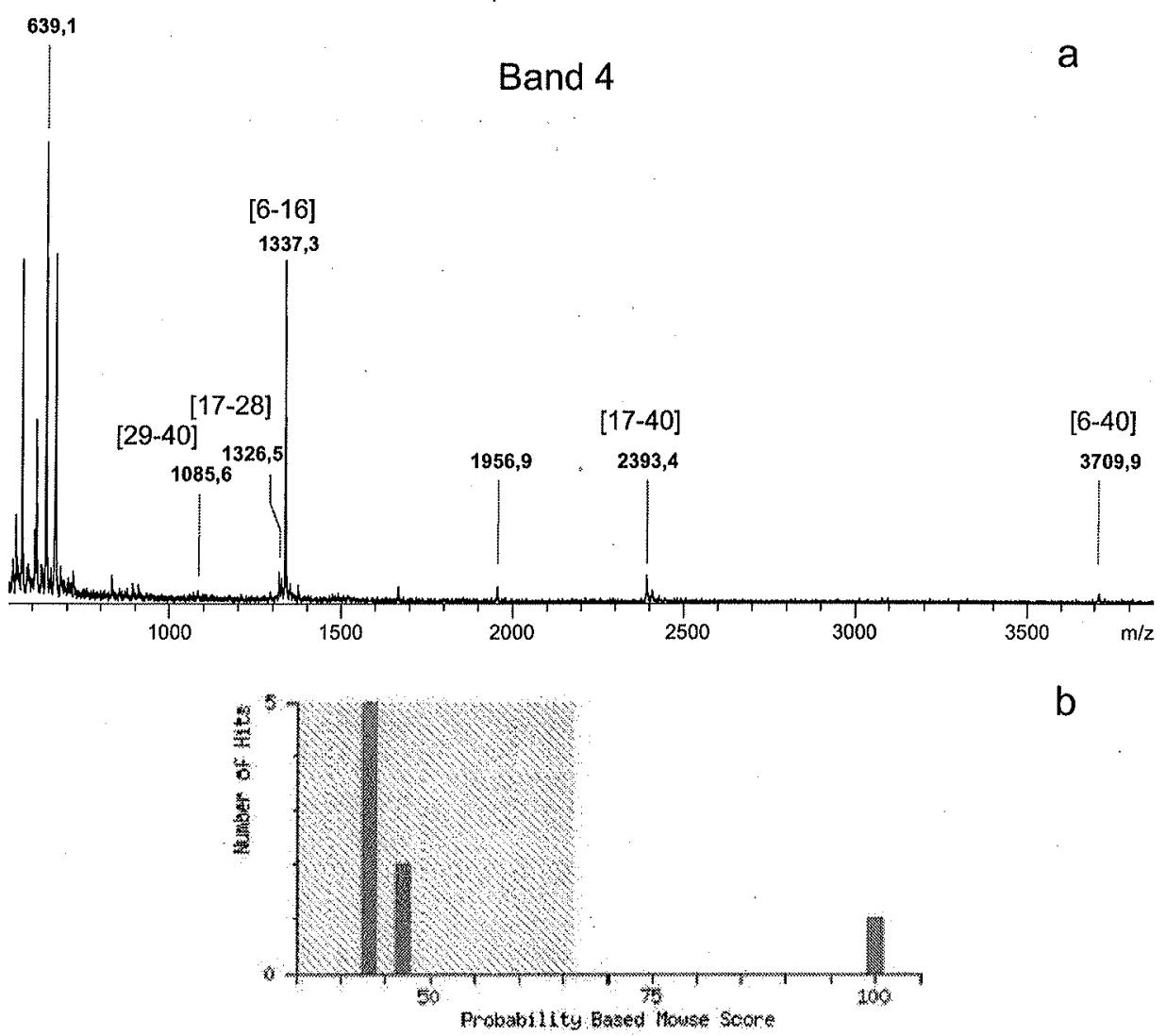

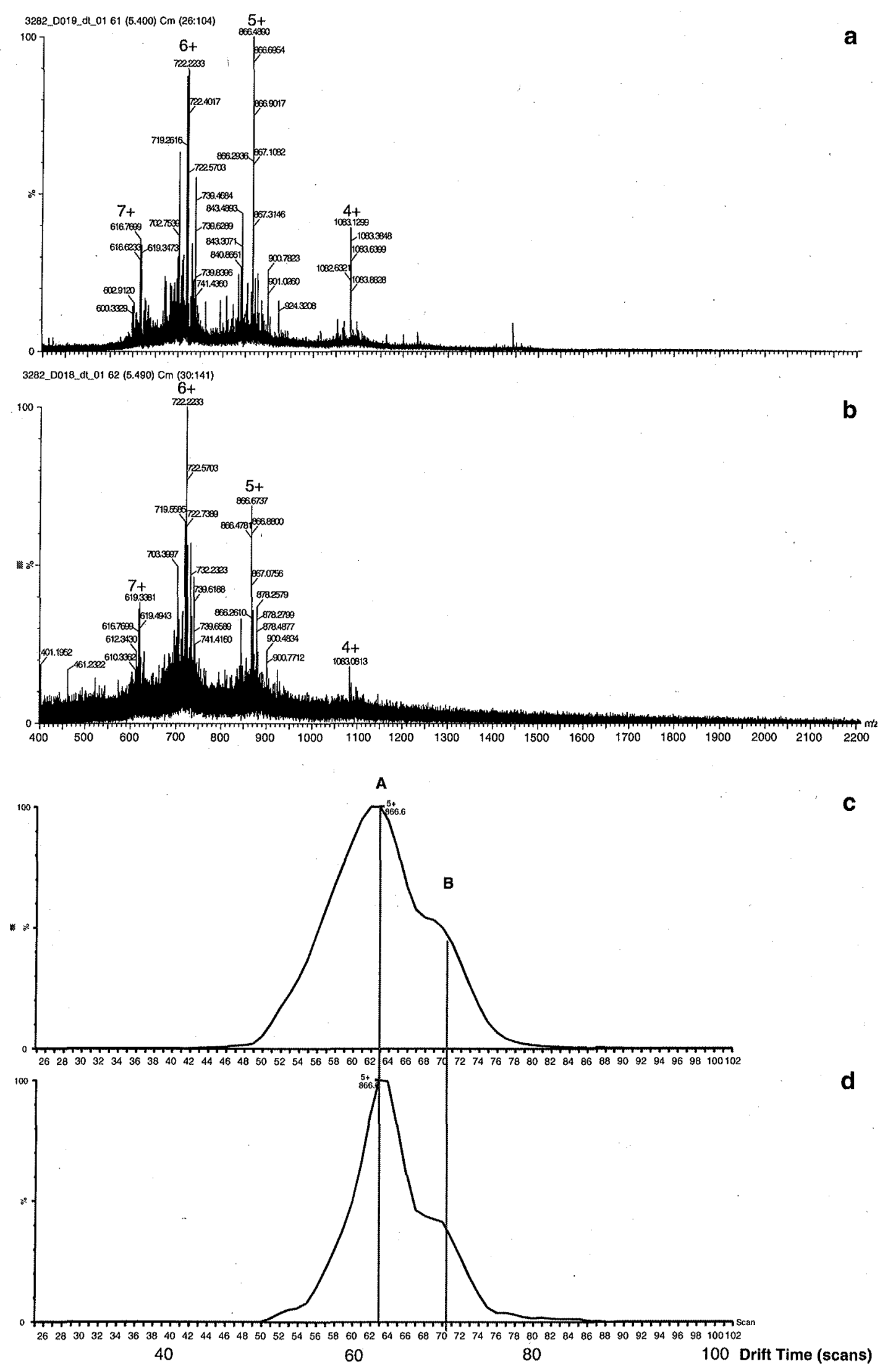
directly for alkylation with IPSL, in order to minimize oxidation and disulfide bridge formation at the $\mathrm{N}$-terminal cysteine residue. To prevent disulfide formation, the IPSL derivative was used at a tenfold molar excess compared to the $A ß$-peptide; at these conditions no dimeric $A ß$-peptide derivatives were observed. The HPLC profile showed the presence of two peaks at 13.6 and $21.5 \mathrm{~min}$, respectively (Fig. 1b); however, MALDI-MS analyses confirmed the presence of only one $A B$-peptide at $21.5 \mathrm{~min}$ due to the IPSL-Cys-A $\beta(1-40)$, while the compound at a retention time of $13.6 \mathrm{~min}$ was found to be unreacted spin-label reagent.

The HPLC-purified peptides, $A B(1-40)$, Cys-Aß $(1-40)$, and IPSL-Cys-Aß $(1-40)$ were also subjected to LC-MS analysis (Fig. 2) which confirmed the expected molecular masses. In all cases, the multiply charged molecular ions $(6+$ and $7+$ ) were found to be the most abundant ions, with loss of one water molecule as the only detectable fragmentation.

Gel electrophoresis and mass spectrometric characterization of in vitro $A ß$-oligomer formation

Due to the insolubility of $A ß$-aggregates, fibril formation cannot be studied directly by mass spectrometry. In order to determine the molecular composition of aggregates, in-gel tryptic digestion and mass spectrometric analysis of the $A B$-oligomeric gel bands was performed. $A B(1-40)$ was solubilized at $1 \mu \mathrm{g} / \mu \mathrm{L}(220 \mu \mathrm{M})$ in fibril growth buffer solution, pH 7.5 and incubated for 5 days at $37^{\circ} \mathrm{C}$. At the end of the incubation, fibrils were obtained as a white precipitate, sonicated, and centrifuged for $15 \mathrm{~min}$, as described in the "Materials and methods" section, and the supernatant was separated from the precipitate for ion mobility-MS. The precipitate was separated from the supernatant, resuspended in MilliQ water, and subjected to polyacrylamide gel electrophoresis (Fig. 3). Only the fraction soluble in running buffer entered the gel (Fig. 3a) while the insoluble part remained at the top of the stacking gel (data not shown). This soluble fraction was found by MALDI-MS to contain monomeric Aß (Fig. 3a, band 1), $\mathrm{A} ß$-oligomers at approximately $20 \mathrm{kDa}$ (pentamer, Fig. 3a-band 2), and high-molecular-weight aggregates at $>100 \mathrm{kDa}$ (Fig. 3a, bands 3 and 4). The Aß-oligomers in Fig. 3a were not separated by SDS-PAGE, but provided separation into defined $A B$-oligomers (dimers to pentamers) in Tris-tricine PAGE (data not shown). All gel bands were cut out, digested with Trypsin and analyzed by MALDIMS. The mass spectrometric data ascertained the presence of $A B$-peptides in all gel bands by the corresponding peptide fragments (Table 1 and Fig. 4).

The preparation of aggregates of the spin-labeled IPSLCys- $A B(1-40)$ peptide was carried out at identical con- ditions, by incubation of a $200 \mu \mathrm{M}$ solution for 5 days at $37^{\circ} \mathrm{C}$. Following incubation, supernatant and precipitate were separated by centrifugation and the precipitate suspended in MilliQ water for gel electrophoresis. Tristricine PAGE analysis was performed with a freshly prepared IPSL-Cys-Aß $(1-40)$ solution in fibril growth buffer, the supernatant and the fibril suspension of the spin-labeled $A B$-aggregates (Fig. 3b). The gel showed that the IPSL-Cys-AB(1-40) peptide started to aggregate already during the solubilization, as indicated by dimeric and trimeric gel bands (Fig. 3b, lane 1). From the fibril precipitate, only the fraction soluble in running buffer entered the separation gel (Fig. 3b), the insoluble part remaining at the top of the stacking gel (data not shown). In the gel, the presence of monomer, dimer, and trimer, and some high-molecular-weight aggregates with a molecular

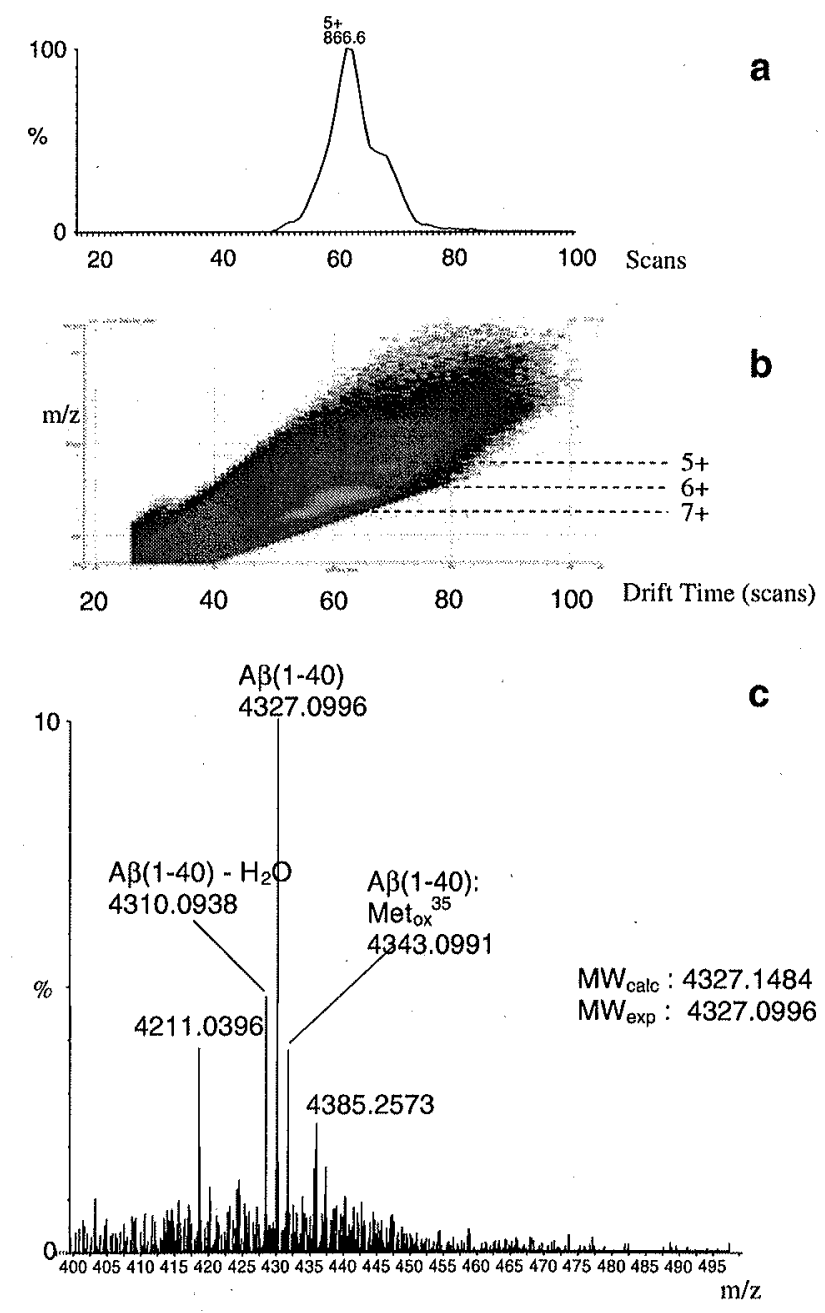

Fig. 6 Drift-time profile of the $A B(1-40)$ supernatant after 5 days of incubation. a The ion mobility profile for the $[\mathrm{M}+5 \mathrm{H}]^{5+}$ ion; $\mathbf{b}$ the drift scope and $\mathbf{c}$ deconvoluted spectrum showing the presence of the Met-35 sulfoxide oxidation product 


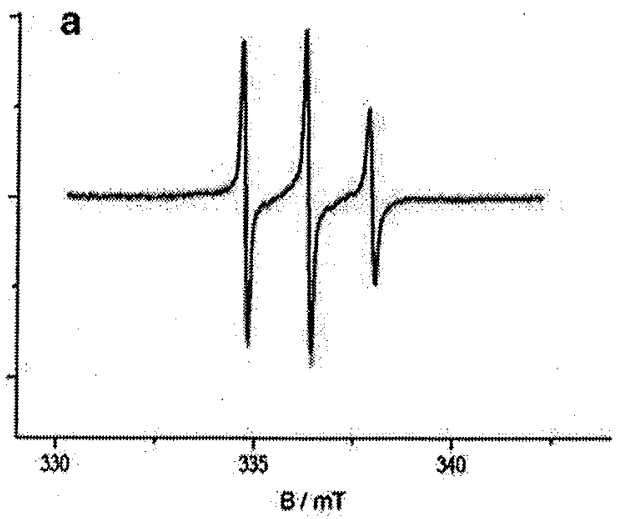

b
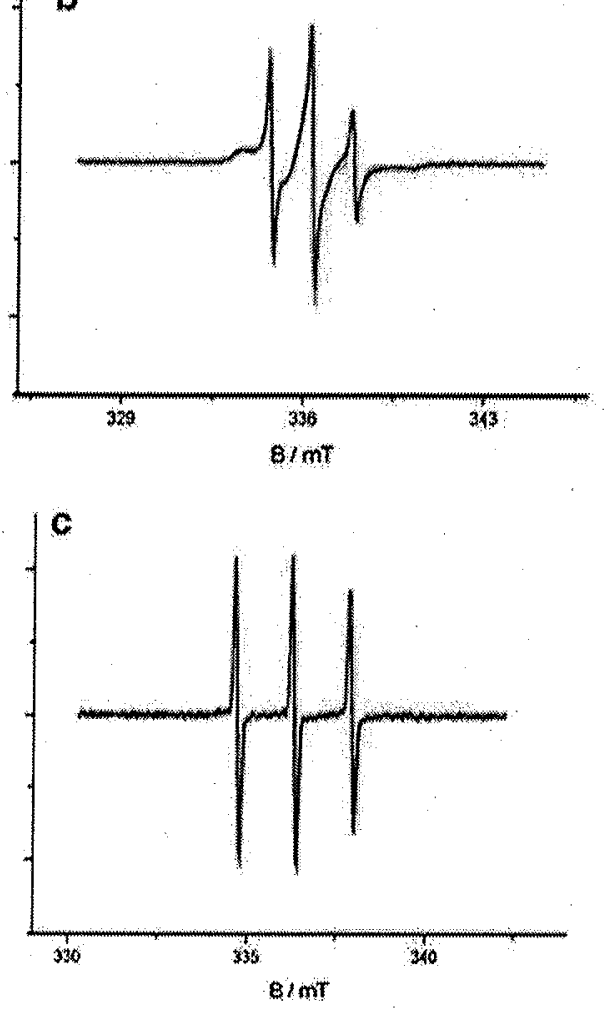

Fig. 7 EPR spectra of a freshly prepared $A B(1-40)$, $b$ the precipitate, and $\mathrm{c}$ the soluble fraction (supernatant) after 5 days of incubation (gray) and corresponding simulations (black; see Table 2 and "Materials and methods" section for parameters) weight beyond $200 \mathrm{kDa}$ were observed (Fig. 3b, lane 2). In contrast, the supernatant showed only the band of the $A B$ monomer (Fig. 3b, lane 3).

Characterization of Aß-oligomers by ion mobility mass spectrometry

The soluble fraction of the $A ß(1-40)$ fibril preparation, obtained by $A B$-incubation for 5 days at $37^{\circ} \mathrm{C}$, and a freshly prepared $A B(1-40)$. peptide solution $(220 \mu \mathrm{M})$ were subjected to comparative analysis by ion mobility-MS (Fig. 5a and b). In the freshly prepared $A ß(1-40)$ solution, the $[\mathrm{M}+5 \mathrm{H}]^{5+}$ ion was found predominant, while in the fibril preparation the $[\mathrm{M}+6 \mathrm{H}]^{6+}$ ion was most abundant. The signal/noise ratio of the $[\mathrm{M}+5 \mathrm{H}]^{5+t}$ and $[\mathrm{M}+6 \mathrm{H}]^{6+}$ ions was lower in the fibril preparation sample than in the freshly prepared $A B(1-40)$ sample, suggesting a lower amount of $A ß(1-40)$ monomer due to the aggregate formation. The extracted ion mobility profiles for the $[\mathrm{M}$ $+5 \mathrm{H}]^{5+}$ ion, of the freshly prepared $A B(1-40$; Fig. $5 \mathrm{c})$ and the fibril preparation (Fig. $5 \mathrm{~d}$ ) indicate the presence of two conformational states (A and $B$ ), with different ion mobilities, which may be indicative of the oligomerization process.

The extracted ion mobility plot for the $[\mathrm{M}+5 \mathrm{H}]^{5+}$ ion of the fibril preparation is presented in Fig. $6 a, b$ together with the drift scope of the 5+, 6+, and 7+ ions. The deconvoluted spectrum of the fibril preparation is shown in Fig. 6c. From the mass spectrometric data of the ion mobility profiles, modifications of $A \beta$ by oxidation and loss of one water molecule could be identified, in addition to the intact $\mathrm{AB}(1-40)$ monomer. The oxidation at the $\mathrm{Met}^{35}$ residue of $A B$ was confirmed by MS/MS sequence determination (data not shown).

Characterization of spin-labeled AB-aggregates by electron paramagnetic resonance spectroscopy

First EPR spectroscopic determinations of the spin-labeled $A B$ monomer peptide, in comparison with the $A ß$-fibril preparations, were performed with the aim to obtain information about the size of $A ß$-aggregates monitored by the local spin-label mobilities. EPR spectra of the freshly

Table 2 Fractions contributing to the EPR spectra as derived from simulation parameters (cf. Fig. 7)

\begin{tabular}{lllll}
\hline & Component 1: 0.036 ns & Component 2: b0.394 ns & Component 3: 0.803 ns & Component 4: 5.46 ns \\
\hline Freshly prepared (7a) & 0.19 & 0.30 & 0.51 & 0.00 \\
Precipitate (7b) & 0.01 & 0.02 & 0.21 & 0.76 \\
Supernatant (7c) & 1.00 & 0.00 & 0.00 & 0.00 \\
\hline
\end{tabular}

For each fraction the rotational correlation time is given 
prepared solution of IPSL-Cys-Aß $(1-40)$, the precipitate residue and the soluble $A B$ - fibril preparation are compared in Fig. $7 \mathrm{a}-\mathrm{c}$ ). All spectra were quantitatively analyzed by spectral-lineshape-simulations. The supernatant spectrum (Fig. 7c) could be described by a single component, the other spectra consist of at least two additional components which were significantly broadened relative to the supernatant spectrum. This broadening reflects the reduced mobility of the spin-label-side group due to oligomerization and/or aggregation.

Using a multi-component simulating strategy a number of spectral components was chosen in accordance with the gel electrophoretic results in order to simulate the spectra. The fractions of the contributing components as well as their rotational correlation times could be identified (Table 2). The supernatant of the fibril preparation sample was found to contain only one component which could be attributed to the $A ß$-monomer, again in agreement with the gel electrophoresis data (Fig. 3b, lane 3). In the freshly prepared IPSL-Cys-Aß $(1-40)$ solution, the corresponding, $A ß$-monomer component was found only with a relative amount of approximately $19 \%$. In addition, two further contributions presumably representing oligomers were found. In the fibril precipitate, the identical mixture of components was found, but the main component $(76 \%)$ featured a very slow rotational correlation time suggesting that this component can be attributed to fibrils. Thus, the EPR data were in complete agreement with the gel electrophoretic separation of $A B$-aggregates, suggesting that aggregation starts rapidly upon solubilization of monomeric $A ß$-peptide in the fibril growth buffer. In the precipitate, almost exclusively high-molecular-weight compounds were found.

\section{Conclusions}

In the present study, ion mobility-MS in combination with electron paramagnetic resonance spectroscopy was explored for the characterization of the oligomerization and fibril formation reaction of $\beta$-amyloid in vitro. The results obtained here indicate the combination of both methods as an efficient tool to characterize the composition of oligomerization products, and the molecular dimensions of aggregate products. The EPR spectra and gel electrophoresis data were in complete agreement to show that (a) $A B$ - aggregation in vitro begins rapidly upon solubilization of the $A B-$ monomer, and (b) the insoluble fibril product contains large aggregates; while in the supernatant, the soluble Aß-monomer is predominant. The ion mobility-MS data provide evidence of (a) the presence of at least two different conformational forms involved in $A \beta$-aggregation, and (b) the formation of a $\mathrm{Met}^{35}$ oxidized $\mathrm{AB}$ peptide during the oligomerization and aggregation. Hence, the complementarity of both methods appears promising for the characterization of the Aß-oligomerization and aggregation pathway. Corresponding studies using suitably modified Aß-peptides is presently carried out in our laboratory.

Acknowledgments We thank Marilena Manea and Marcel Leist for expert help with the synthesis of spin-labeled AB-peptide derivatives, and critical discussion of the manuscript, and Martin Spitzbarth for help with the EPR simulations. This work was supported by the International Research Center "Proteostasis" at the University of Konstanz, and by the Deutsche Forschungsgemeinschaft, Bonn, Germany (DR 743/2-1).

\section{References}

1. Jakobsen LD, Jensen PH (2003) Methods Mol Biol 232:57-66

2. Morgan D (2006) J Alzheimers Dis 9:425-432

3. Uversky VN (2007) J Neurochem 103:17-37

4. Salminen A, Ojala J, Kauppinen A, Kaarniranta K, Suuronen T (2009) Prog Neurobiol 87:181-194

5. Crews L, Tsigelny I, Hashimoto M, Masliah E (2009) Neurotox. Res in press

6. Hull M, Berger M, Heneka M (2006) Drugs 66:2075-2093

7. Gardberg AS, Dice LT, Ou S, Rich RL, Helmbrecht E, Ko J Wetzel R, Myszka DG, Patterson PH, Dealwis C (2007) Proc Natl Acad Sci USA 104:15659-15664

8. Luhrs T, Ritter C, Adrian M, Riek-Loher D, Bohrmann B, Dobeli H, Schubert D, Riek R (2005) Proc Natl Acad Sci USA 102:17342-17347

9. McLaurin J, Cecal R, Kierstead ME, Tian X, Phinney AL, Manea M, French JE, Lambermon MH, Darabie AA, Brown ME, Janus C, Chishti MA, Horne P, Westaway D, Fraser PE, Mount HT, Przybylski M, St George-Hyslop P (2002) Nature Med 8:1263-1269

10. Solomon B (2007) Drugs Today (Barc) 43:333-342

11. Grau S, Baldi A, Bussani R, Tian X, Stefanescu R, Przybylski M, Richards P, Jones SA, Shridhar V, Clausen T, Ehrmann M (2005) Proc Natl Acad Sci USA 102:6021-6026

12. Dodel R, Hampel H, Depboylu C, Lin S, Gao F, Schock S, Jackel S, Wei X, Buerger K, Hoft C, Hemmer B, Moller HJ, Farlow M, Oertel WH, Sommer N, Du Y (2002) Ann Neurol 52:253-256

13. Stefanescu R, Iacob RE, Damoc EN, Marquardt A, Amstalden E, Manea M, Perdivara I, Maftei M, Paraschiv G, Przybylski M (2007) Eur J Mass Spectrom (Chichester, Eng) 13:69-75

14. Manea M, Hudecz F, Przybylski M, Mezo G (2005) Bioconj Chem 16:921-928

15. Perdivara I, Deterding LJ, Cozma C, Tomer KB, Przybylski M (2009) Glycobiology, in press

16. Juszczyk P, Paraschiv G, Szymanska A, Kolodziejczyk AS, Rodziewicz-Motowidlo S, Grzonka Z, Przybylski M (2009) J Med Chem 52:2420-2428

17. Ruotolo BT, Benesch JL, Sandercock AM, Hyung SJ, Robinson CV (2008) Nature Prot 3:1139-1152

18. Ruotolo BT, Hyung SJ, Robinson PM, Giles K, Bateman RH, Robinson CV (2007) Angew Chem Int Ed Engl 46:80018004

19. Kanu AB, Dwivedi P, Tam M, Matz L, Hill HH Jr (2008) J Mass Spectrom 43:1-22

20. Trimpin S, Clemmer DE (2008) Anal Chem 80:9073-9083 
21. Henderson SC, Valentine SJ, Counterman AE, Clemmer DE (1999) Anal Chem 71:291-301

22. Hoaglund CS, Valentine SJ, Sporleder CR, Reilly JP, Clemmer DE (1998) Anal Chem 70:2236-2242

23. Zhou M, Sandercock AM, Fraser CS, Ridlova G, Stephens E, Schenauer MR, Yokoi-Fong T, Barsky D, Leary JA, Hershey JW, Doudna JA, Robinson CV (2008) Proc Natl Acad Sci USA 105:18139-18144

24. Drescher M, Godschalk F, Veldhuis $G$, van Rooijen $B D$, Subramaniam V, Huber M (2008) Chembiochem 9:2411-2416

25. Drescher $M$, Veldhuis $G$, van Rooijen $B D$, Milikisyants $S$, Subramaniam V, Huber M (2008) J Am Chem Soc 130:7796-7797
26. Murakami K, Hara H, Masuda Y, Ohigashi H, Irie K (2007) Chembiochem 8:2308-2314

27. Torok M, Milton S, Kayed R, Wu P, McIntire T, Glabe CG, Langen R (2002) J'Biol Chem 277:40810 40815

28. Zager SA, Freed JH (1982) J Chem Phys 77:3344-3349

29. Le Meste M, Voilley A (1988) J Phys Chem 92:1612-1616

30. Jeschke $G$ (2002) ChemPhysChem 3:927-932

31. Kirby TL, Karim CB, Thomas DD (2004) Biochemistry 43:58425852

32. Gettins P, Beth AH, Cunningham LW (1988) Biochemistry 27:2905-2911

33. Stoll S, Schweiger A (2006) J Magnet Resonance 178:42--55 